\title{
Wavelength-selective fluorescence in ion channels formed by gramicidin $\mathbf{A}$ in membranes
}

\author{
AMITABHA CHATTOPADHYAY* and SATINDER S RAWAT ${ }^{\#}$ \\ Centre for Cellular and Molecular Biology, Uppal Road, Hyderabad 500007 \\ "Present address: 364 Plantation Street, Room 570R, Lazare Research Building, \\ University of Massachusetts Medical School, Worcester, MA 01605, USA \\ e-mail: amit@ccmb.res.in
}

\begin{abstract}
Gramicidins are linear peptides that form ion channels that are specific for monovalent cations in membranes. The tryptophan residues in the gramicidin channel play a crucial role in the organization and function of the channel. The natural mixture of gramicidins, denoted as gramicidin $\mathrm{A}^{\prime}$, consists of mostly gramicidin A, but also contains gramicidins B, C and D as minor components. We have previously shown that the tryptophan residues in ion channels formed by the naturally occurring peptide, gramicidin $\mathrm{A}^{\prime}$, display wavelength-dependent fluorescence characteristics due to the motionally restricted environment in which they are localized. In order to check the influence of ground-state heterogeneity in the observed wavelength-selective fluorescence of gramicidin $\mathrm{A}^{\prime}$ in membranes, we performed similar experiments with pure gramicidin A in model membranes. Our results show that the observed wavelength-selective fluorescence characteristics of naturally occurring gramicidin $\mathrm{A}^{\prime}$ are not due to groundstate heterogeneity.
\end{abstract}

Keywords. Gramicidins; ion channels; wavelength-dependent fluorescence; wavelength-selective fluorescence; red-edge excitation shift.

\section{Introduction}

The linear peptide gramicidin forms prototypical ion channels that are specific for monovalent cations and has been extensively used to study the organization, dynamics and function of membrane-spanning channels. ${ }^{1-3}$ The transmembrane gramicidin channel is formed by the head-to-head dimerization of $\beta^{6.3}$ helices. ${ }^{4}$ The channel interior is lined by the polar carbonyl and amide moieties of the peptide backbone, a feature shared with the selectivity filter of the bacterial $\mathrm{KcsA} \mathrm{K}^{+}$channel. ${ }^{5}$ An important aspect of this conformation is the membrane interfacial location of the tryptophan residues, a common feature of many transmembrane helices. ${ }^{6-8}$

Gramicidins are linear pentadecapeptide antibiotics with a molecular weight of $\sim 1900$. They are produced by the soil bacterium Bacillus brevis, and consist of alternating L- and D-amino acids. ${ }^{9}$ The natural mixture of gramicidins, often denoted as gramicidin $\mathrm{A}^{\prime}$ (termed as gramicidin $\mathrm{D}$ in older literature), consists of gramicidin A (80-85\%), gramicidin B (6-7\%), gramicidin C (5-14\%), and gramicidin D

*For correspondence
$(1 \%) .{ }^{10}$ The various types of gramicidins differ in one residue. Gramicidin A and D have four tryptophan residues at positions $9,11,13$ and 15 (see table 1). However, the Trp-11 in gramicidin A and D is replaced by $\mathrm{Phe}$ in gramicidin $\mathrm{B}$, and Tyr in gramicidin C. On the other hand, the Gly-2 of gramicidin A is replaced by Ala in gramicidin D. Gramicidin A' is readily available commercially and is fluorescent, due to the presence of tryptophan residues. ${ }^{11,12}$ It has one of the most hydrophobic sequences known and has been widely used as a model peptide for membrane-spanning regions of intrinsic membrane proteins. ${ }^{13,14}$

The tryptophan residues in gramicidin channels are believed to be crucial for maintaining the structure and function of the channel. ${ }^{15-17}$ We have earlier performed wavelength-selective fluorescence experiments using the tryptophan residues in the channel conformation of the naturally occurring peptide, gramicidin $\mathrm{A}^{\prime}{ }^{11,18}$ Wavelength-selective fluorescence comprises a set of approaches based on the red-edge effect in fluorescence spectroscopy, which can be used to directly monitor the environment and dynamics around a fluorophore in a complex biological system. A shift in the wavelength of maximum fluorescence 
Table 1. Amino acid sequences of various gramicidin peptides. The differences in sequence among the peptides and positions of the tryptophan residues are highlighted.

\begin{tabular}{|c|c|c|}
\hline $\begin{array}{l}\text { Gramicidin } \\
\text { type }\end{array}$ & Amino acid sequence & $\begin{array}{l}\text { Percent content } \\
\text { in gramicidin } \mathrm{A}^{\prime}\end{array}$ \\
\hline A & $\begin{array}{l}\text { HCO-L-Val-Gly-L-Ala-D-Leu-L-Ala-D-Val-L-Val-D-Val-L-Trp-D-Leu-L-Trp-D-Leu-L- } \\
\text { Trp-D-Leu-L-Trp-NHCH}{ }_{2} \mathrm{CH}_{2} \mathrm{OH} \\
13 \\
15\end{array}$ & $80-85 \%$ \\
\hline B & $\begin{array}{l}\text { HCO-L-Val-Gly-L-Ala-D-Leu-L-Ala-D-Val-L-Val-D-Val-L-Trp-D-Leu-L-Phe-D-Leu-L- } \\
\text { Trp-D-Leu-L-Trp-NHCH } \mathrm{CH}_{2} \mathrm{OH} \\
13\end{array}$ & $6-7 \%$ \\
\hline $\mathrm{C}$ & $\begin{array}{l}\text { HCO-L-Val-Gly-L-Ala-D-Leu-L-Ala-D-Val-L-Val-D-Val-L-Trp-D-Leu-L-Tyr-D-Leu-L- } \\
\text { Trp-D-Leu-L-Trp-NHCH } \mathrm{CH}_{2} \mathrm{OH} \\
13\end{array}$ & $5-14 \%$ \\
\hline $\mathrm{D}$ & $\begin{array}{l}\text { HCO-L-Val-D-Ala-L-Ala-D-Leu-L-Ala-D-Val-L-Val-D-Val-L-Trp-D-Leu-L-Trp-D-Leu-L- } \\
\text { Trp-D-Leu-L-Trp-NHCH}{ }_{2} \mathrm{CH}_{2} \mathrm{OH} \\
13\end{array}$ & $1 \%$ \\
\hline
\end{tabular}

emission toward higher wavelengths, caused by a shift in the excitation wavelength toward the rededge of the absorption band, is termed red edge excitation shift (REES). This effect is mostly observed with polar fluorophores in motionally restricted media such as very viscous solutions or condensed phases, where the dipolar relaxation time for the solvent shell around a fluorophore is comparable to or longer than its fluorescence lifetime. ${ }^{19-23}$ REES arises from slow rates of solvent relaxation (reorientation) around an excited-state fluorophore which depends on the motional restriction imposed on the solvent molecules in the immediate vicinity of the fluorophore. Utilizing this approach, it becomes possible to probe the mobility parameters of the environment itself (which is represented by the relaxing solvent molecules) using the fluorophore merely as a reporter group. Further, since the ubiquitous solvent for biological systems is water, the information obtained in such cases would come from the otherwise 'optically silent' water molecules.

We have previously shown that the tryptophan residues in channels formed by the naturally occurring peptide, gramicidin $\mathrm{A}^{\prime}$, exhibit REES due to the motionally restricted environment in which they are localized in this conformation in the membrane interface. ${ }^{11,18}$ Since gramicidin $\mathrm{A}^{\prime}$ is not a chemically pure compound and is a mixture of peptides with some peptides (gramicidin B and C) having different number of tryptophan residues, the observed REES from channels formed by gramicidin $\mathrm{A}^{\prime}$ could be due to ground-state heterogeneity. In this paper, we have addressed this issue by performing wavelengthselective fluorescence experiments on the channel conformation formed by pure gramicidin A in model membranes.

\section{Materials and methods}

\subsection{Materials}

1-Palmitoyl-2-oleoyl-sn-glycero-3-phosphocholine (POPC) was obtained from Avanti Polar Lipids (Alabaster, AL, USA). Pure gramicidin A was purified from commercial gramicidin $\mathrm{A}^{\prime}$ (from Bacillus brevis) and was a generous gift from Prof. Roger $\mathrm{E}$ Koeppe (University of Arkansas, USA). Dimyristoyl-sn-glycero-3-phosphocholine (DMPC) was purchased from Sigma Chemical Co. (St. Louis, MO, USA). Lipids were checked for purity by thin layer chromatography on silica gel precoated plates (Sigma) in chloroform/methanol/water $(65: 35: 5$, $\mathrm{v} / \mathrm{v} / \mathrm{v}$ ) and were found to give only one spot in all cases with a phosphate-sensitive spray and subsequent charring. ${ }^{24}$ The concentration of POPC was determined by phosphate assay subsequent to total digestion by perchloric acid. ${ }^{25}$ DMPC was used as an internal standard to assess lipid digestion. All other chemicals used were of the highest purity available. Solvents used were of spectroscopic grade. Water was purified through a Millipore (Bedford, MA, USA) Milli-Q system and used for all experiments. 


\subsection{Sample preparation}

All experiments were done using unilamellar vesicles (ULV) of POPC containing $2 \%(\mathrm{~mol} / \mathrm{mol})$ gramicidin A. The channel conformation of gramicidin A was generated essentially as described earlier. ${ }^{11}$ In general, $1280 \mathrm{nmol}$ of POPC in chloroform was mixed with $25.6 \mathrm{nmol}$ of gramicidin A in methanol. A few drops of chloroform were added and mixed well, and the samples were dried under a stream of nitrogen while being warmed gently $\left(\sim 35^{\circ} \mathrm{C}\right)$. After further drying under high vacuum for at least $3 \mathrm{~h}$, $3 \mathrm{ml}$ of $10 \mathrm{mM}$ sodium phosphate, and $150 \mathrm{mM}$ of sodium chloride buffer, $\mathrm{pH} 7 \cdot 0-7 \cdot 2$, was added, and each sample vortexed for 3 min to disperse the lipid. The lipid dispersions so obtained were sonicated for $10 \mathrm{~min}$ (in bursts of $1 \mathrm{~min}$, followed by immediate cooling in ice) using a Branson model 250 sonifier fitted with a microtip. The sonicated samples were centrifuged at $15,000 \mathrm{rpm}$ for $20 \mathrm{~min}$ to remove titanium particles shed from the microtip during sonication and incubated overnight at $65^{\circ} \mathrm{C}$ with continuous shaking in order to induce channel conformation. ${ }^{26,27}$ Background samples were prepared the same way except that gramicidin was omitted. All experiments were done with multiple sets of samples at $25^{\circ} \mathrm{C}$.

\subsection{Steady state fluorescence measurements}

Steady state fluorescence measurements were performed with a Hitachi F-4010 steady state spectrofluorometer using $1-\mathrm{cm}$ path length quartz cuvettes. Excitation and emission slits with a nominal bandpass of $5 \mathrm{~nm}$ were used. Background intensities of samples in which gramicidin A was omitted were subtracted from each sample spectrum to cancel out any contribution due to the solvent Raman peak and other scattering artifacts. The spectral shifts obtained with different sets of samples were identical in most cases. In other cases, the values were within $\pm 1 \mathrm{~nm}$ of the ones reported. Fluorescence polarization measurements were performed using a Hitachi polarization accessory. Polarization values were calculated from the equation, ${ }^{28}$

$$
P=\left(I_{\mathrm{VV}}-G I_{\mathrm{VH}}\right) /\left(I_{\mathrm{VV}}+G I_{\mathrm{VH}}\right),
$$

where $I_{\mathrm{Vv}}$ and $I_{\mathrm{VH}}$ are the measured fluorescence intensities (after appropriate background subtraction) with the excitation polarizer vertically oriented and emission polarizer vertically and horizontally oriented, respectively. $G$ is the grating correction factor and is the ratio of the efficiencies of the detection system for vertically and horizontally polarized light, and is equal to $I_{\mathrm{HV}} / I_{\mathrm{HH}}$. All experiments were done with multiple sets of samples and average values of polarization are shown in figures 3 and 4 .

\subsection{Circular dichroism measurements}

CD measurements were carried out at room temperature $\left(25^{\circ} \mathrm{C}\right)$ on a Jasco $\mathrm{J}-715$ spectropolarimeter which was calibrated with $(+)$-10-camphorsulphonic acid. ${ }^{29}$ The spectra were scanned in a quartz optical cell with a path length of $0 \cdot 1 \mathrm{~cm}$. All spectra were recorded in $0.5 \mathrm{~nm}$ wavelength increments with a $4 \mathrm{~s}$ response and a band width of $1 \mathrm{~nm}$. For monitoring changes in secondary structure, spectra were scanned in the far-UV range from 200 to $280 \mathrm{~nm}$ at a scan rate of $100 \mathrm{~nm} / \mathrm{min}$. Each spectrum is the average of 12 scans with full-scale sensitivity of $10 \mathrm{mdeg}$. All spectra were corrected for background by subtraction of appropriate blanks and were smoothed making sure that the overall shape of the spectrum remains unaltered. Data are represented as mean residue ellipticities and were calculated using the formula:

$$
[\theta]=\theta_{\text {obs }} /(10 C l),
$$

where $\theta_{\text {obs }}$ is the observed ellipticity in mdeg, $l$ is the path length in $\mathrm{cm}$, and $C$ is the concentration of peptide bonds in $\mathrm{mol} / \mathrm{L}$.

\section{Results and discussion}

\subsection{Circular dichroism spectroscopy shows that gramicidin A forms channels in membranes}

Circular dichroism spectroscopy has been previously utilized to characterize various membrane-bound conformations of gramicidin. ${ }^{26,27} \mathrm{We}$ therefore used characteristic $\mathrm{CD}$ spectroscopic features to confirm the conformation of gramicidin A in POPC vesicles. The CD spectrum of gramicidin A in POPC vesicles is shown in figure 1. The spectrum for the channel conformation of gramicidin has two characteristic peaks of positive ellipticity around 218 and $235 \mathrm{~nm}$, a valley around $230 \mathrm{~nm}$, and negative ellipticity below $208 \mathrm{~nm}^{26,27}$ These are characteristic of the singlestranded $\beta^{6.3}$ conformation. As shown in figure 1 , the CD spectrum of gramicidin A agrees with this criterion, and is therefore representative of the channel conformation. 


\subsection{Red-edge excitation shift of gramicidin A tryptophans}

Shift in the maxima of fluorescence emission ${ }^{\ddagger}$ of the tryptophan residues of gramicidin A in the channel conformation as a function of excitation wavelength is shown in figure 2. Tryptophans in the channel form of gramicidin A exhibit an emission maximum of $332 \mathrm{~nm}$, when excited at $280 \mathrm{~nm}$, similar to that previously reported for gramicidin $\mathrm{A}^{\prime}{ }^{11,18}$ As the excitation wavelength is changed from 280 to $310 \mathrm{~nm}$, the emission maximum is shifted from 332 to $338 \mathrm{~nm}$, which corresponds to a REES of $6 \mathrm{~nm}$. It is possible that there could be further red shift if excitation is carried out beyond $310 \mathrm{~nm}$. We found it difficult to work in this wavelength range due to low signal-to-noise ratio and artifacts due to the solvent Raman peak that sometimes remained even after background subtraction.

Such dependence of the emission maximum on excitation wavelength is characteristic of the rededge excitation shift. This implies that the tryptophans in the channel conformation of gramicidin $\mathrm{A}$ are localized in a motionally restricted region of the membrane. This is consistent with our earlier findings that the channel tryptophans gramicidin $\mathrm{A}^{\prime}$ are localized at the membrane interface. ${ }^{11,18}$ Interestingly, the REES exhibited by channels formed by gramicidin $\mathrm{A}^{\prime 11,18}$ and gramicidin $\mathrm{A}$ (present results) were found to be essentially the same. This rules out any contribution of ground-state heterogeneity toward the observed REES of gramicidin $\mathrm{A}^{\prime}$.

\subsection{Wavelength-dependent fluorescence polarization of gramicidin A tryptophans}

Fluorescence polarization is known to be dependent on excitation and emission wavelengths in motionally restricted media. ${ }^{21}$ The excitation polarization spectrum (i.e., a plot of steady-state polarization vs. excitation wavelength) of gramicidin A in POPC vesicles is shown in figure 3 . The fluorescence polarization of gramicidin $\mathrm{A}$ in the membrane displays

\footnotetext{
${ }^{\ddagger}$ We have used the term maximum of fluorescence emission in a somewhat wider sense here. In every case, we have monitored the wavelength corresponding to maximum fluorescence intensity, as well as the centre of mass of the fluorescence emission. In most cases, both these methods yielded the same wavelength. In cases where minor discrepancies were found, the centre of mass of emission has been reported as the fluorescence maximum.
}

characteristic change upon increasing the excitation wavelength, with a sharp increase occurring toward the red-edge of the absorption band. Such an increase in polarization upon red-edge excitation for peptides

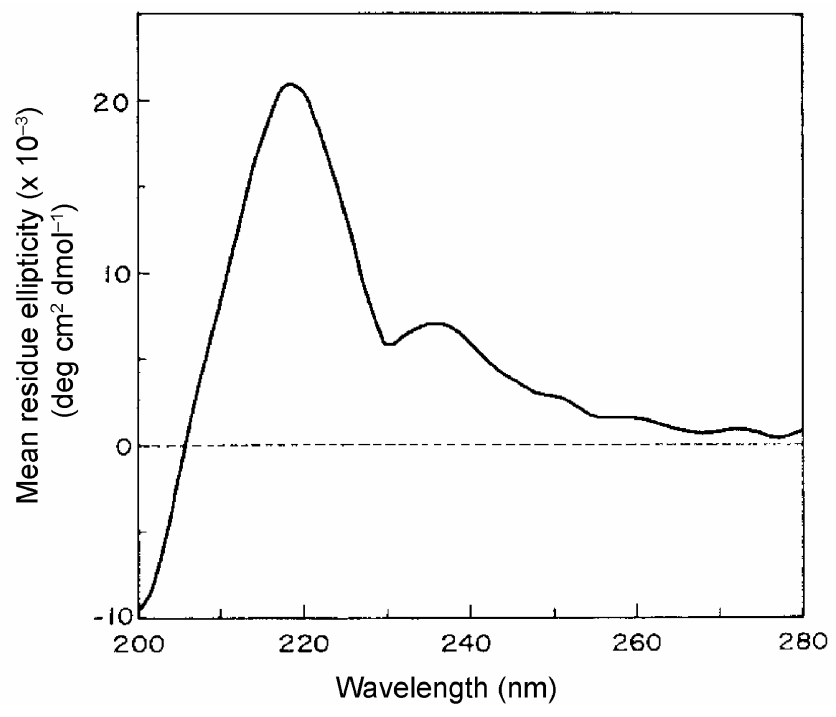

Figure 1. Far-UV CD spectra of the channel form of gramicidin $\mathrm{A}$ in vesicles of POPC. The ratio of gramicidin to POPC was $1: 50(\mathrm{~mol} / \mathrm{mol})$. See $\$ 2$ for other details.

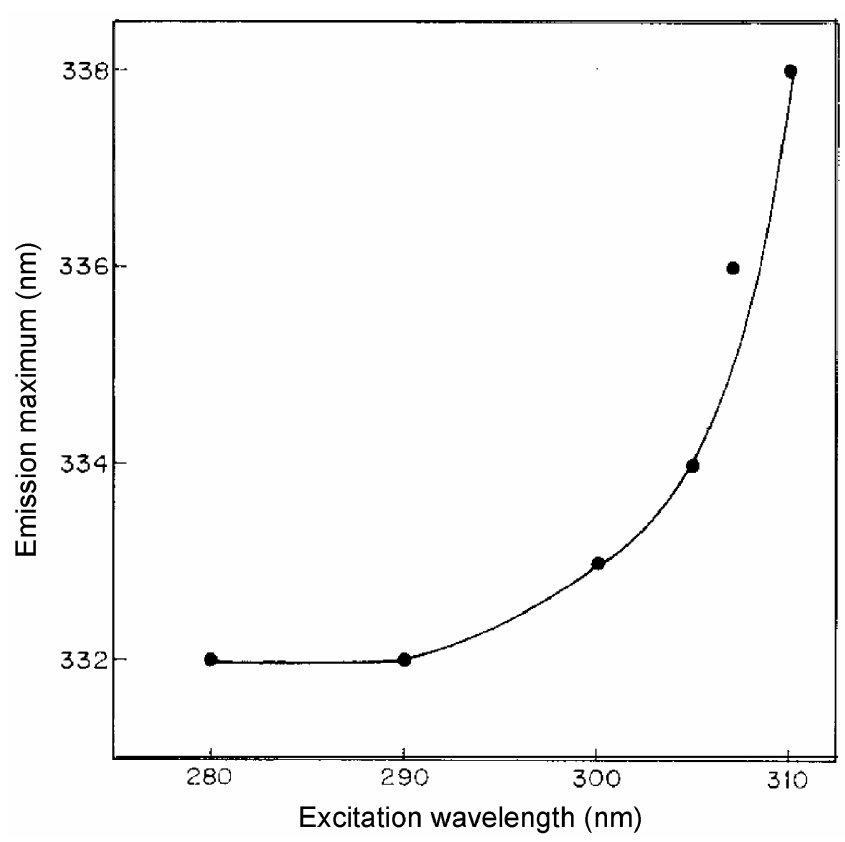

Figure 2. Effect of changing excitation wavelength on the wavelength of maximum emission for the channel form of gramicidin $\mathrm{A}$. The ratio of gramicidin to POPC was $1: 50(\mathrm{~mol} / \mathrm{mol})$. All other conditions are as in figure 1. See $\S 2$ for other details. 
and proteins containing tryptophans in media of reduced mobility has been reported before. ${ }^{21}$ This reinforces that the tryptophan residues in gramicidin A are localized in a motionally restricted region of the membrane. As a control, the fluorescence polarization of gramicidin A in methanol was monitored, which remained essentially invariant over the range of excitation wavelengths. Figure 4 shows the variation in steady-state polarization of tryptophan residues of gramicidin A in POPC vesicles and in methanol, as a function of wavelength across its emission spectrum. As seen from the figure, while polarization values do not show any significant variation over the entire emission range in methanol, there is a considerable decrease in polarization with increasing emission wavelength in case of membrane-bound gramicidin A, as expected for fluorophores in a restricted environmernt. ${ }^{21}$ These wavelength-dependent changes in fluorescence polarization of gramicidin A tryptophans are similar to the changes previously observed with membrane-bound gramicidin $\mathrm{A}^{\prime}{ }^{11}$

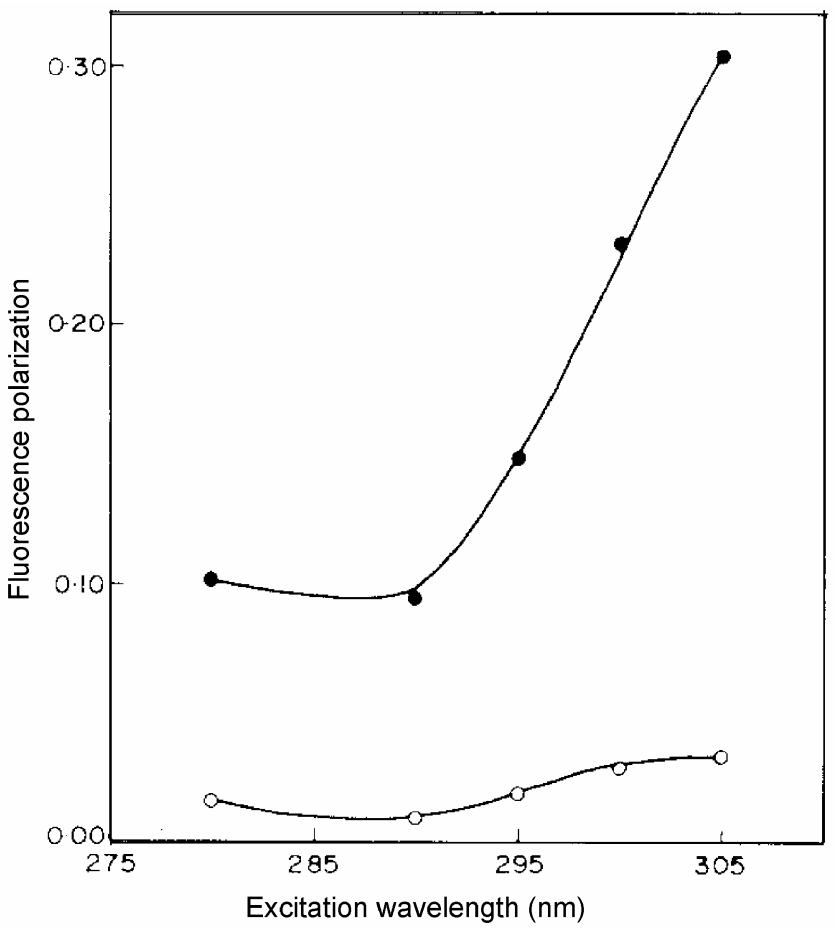

Figure 3. Fluorescence polarization of the channel form of gramicidin A in POPC vesicles (-) as a function of excitation wavelength. Fluorescence polarization of gramicidin A in methanol (O) as a function of excitation wavelength is shown as a control. Polarization values were recorded at $331 \mathrm{~nm}$. The ratio of gramicidin to POPC was $1: 50(\mathrm{~mol} / \mathrm{mol})$. Concentration of gramicidin A in methanol was $8.6 \mu \mathrm{M}$. See $\S 2$ for other details.
Although the functional properties (channel conductance) displayed by gramicidins $A, B$, and $C$ are different, the backbone conformations of these peptides in micelles have previously been found to be essentially identical using NMR spectroscopy. ${ }^{30}$ Interestingly, it has earlier been reported that fluorescence lifetimes of pure gramicidin A in model membranes are identical with those obtained for the commercially available gramicidin $\mathrm{A}^{\prime}$ samples. ${ }^{31} \mathrm{In}$ addition, ground state heterogeneity arising either due to the presence of gramicidin A in the bulk aqueous phase or due to the presence of gramicidin A monomers in the membrane can be ruled out as follows. Contribution from gramicidin A monomer in water is unlikely since gramicidin A is very hydrophobic resulting in extremely low solubility in aqueous solutions $\left(\approx 5 \times 10^{-7} \mathrm{M}\right){ }^{32}$ Also, the dimerization constant of gramicidin A in DOPC black lipid membranes to be $\approx 2 \times 10^{13} \mathrm{~mol}^{-1} \mathrm{~cm}^{2}{ }^{33}$ This means that at the peptide concentration generally employed for spectroscopic experiments in lipid vesicles, the fraction of monomers is negligible. Taken together, our results show that the observed wavelength-selective fluorescence characteristics of naturally occurring gramicidin $\mathrm{A}^{\prime}$, which is a mixture of peptides, is not due to ground-state heterogeneity, since essentially the similar results are obtained when similar

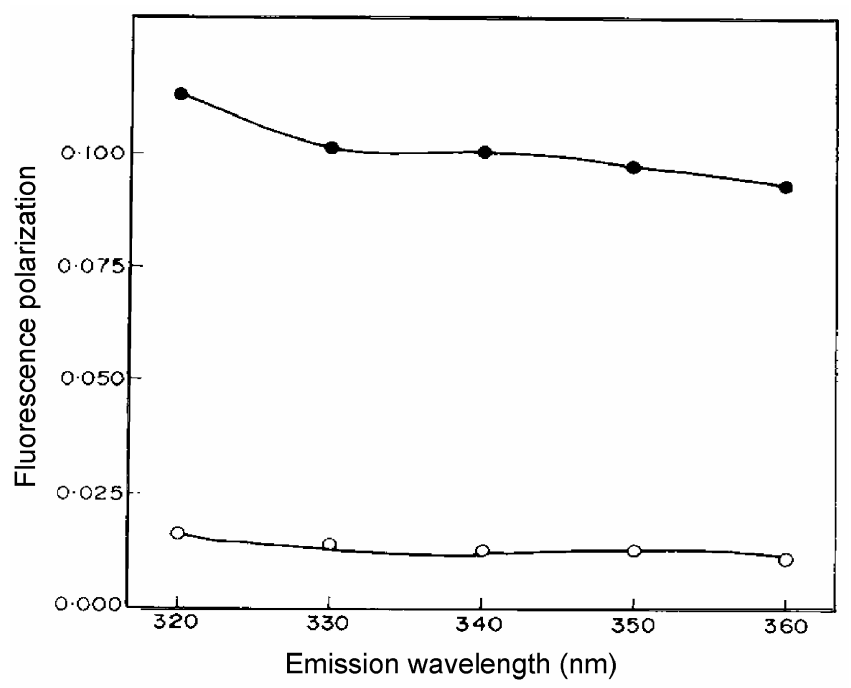

Figure 4. Fluorescence polarization of the channel form of gramicidin A in POPC vesicles ( $)$ as a function of emission wavelength. Fluorescence polarization of gramicidin A in methanol (O) as a function of emission wavelength is shown as a control. The excitation wavelength was $280 \mathrm{~nm}$. The ratio of gramicidin to POPC was $1: 50(\mathrm{~mol} / \mathrm{mol})$. Concentration of gramicidin $A$ in methanol was $8.6 \mu \mathrm{M}$. See $\$ 2$ for other details. 
experiments were performed with chemically pure gramicidin $\mathrm{A}$.

\section{Acknowledgements}

This work was supported by the Department of Science and Technology, and the Council of Scientific and Industrial Research, Government of India. SSR thanks the Council of Scientific and Industrial Research for a fellowship. We gratefully acknowledge Devaki A Kelkar for helpful discussions. We thank Y S S V Prasad and G G Kingi for technical help, and Sourav Haldar for help with the figures. We thank members of our laboratory for critically reading the manuscript.

\section{References}

1. Killian J A 1992 Biochim. Biophys. Acta 1113391

2. Koeppe R E and Andersen O S 1996 Annu. Rev. Biophys. Biomol. Struct. 25231

3. Wallace B A 2000 BioEssays 22227

4. O'Connell A M, Koeppe R E and Andersen O S 1990 Science 2501256

5. Kelkar D A and Chattopadhyay A 2006 J. Biosci. 31297

6. Reithmeier R A F 1995 Curr. Opin. Struct. Biol. 5 491

7. Yau W-M, Wimley W C, Gawrisch K and White S H 1998 Biochemistry 3714713

8. Ulmschneider M B and Sansom M S P 2001 Biochim. Biophys. Acta 15121

9. Sarges R and Witkop B $1965 \mathrm{~J}$. Am. Chem. Soc. 87 2011

10. Abo-Riziq A, Crews B O, Callahan M P, Grace L and de Vries M S 2006 Angew. Chem., Int. Ed. 455166

11. Mukherjee S and Chattopadhyay A 1994 Biochemistry 335089
12. Rawat S S, Kelkar D A and Chattopadhyay A 2005 Biophys. J. 893049

13. Andersen O S, Nielsen C, Maer A M, Lundbæk J A, Goulian M and Koeppe R E 1999 Methods Enzymol. 294208

14. Martinac B and Hamill O P 2002 Proc. Natl. Acad. Sci. USA 994308

15. $\mathrm{Hu} \mathrm{W}$, Lee K-C and Cross T A 1993 Biochemistry 32 7035

16. Salom D, Pérez-Payá E, Pascal J and Abad C 1998 Biochemistry 3714279

17. Andersen O S, Greathouse D V, Providence L L, Becker M D and Koeppe R E 1998 J. Am. Chem. Soc. 1205142

18. Rawat S S, Kelkar D A and Chattopadhyay A 2004 Biophys. J. 87831

19. Demchenko A P 1988 Trends Biochem. Sci. 13374

20. Demchenko A P 2002 Luminescence 1719

21. Mukherjee S and Chattopadhyay A 1995 J. Fluoresc. 5237

22. Chattopadhyay A 2003 Chem. Phys. Lipids 1223

23. Raghuraman H, Kelkar D A and Chattopadhyay A 2005 Reviews in fluorescence (eds) C D Geddes and J R Lakowicz (Springer: New York) vol. 2, pp 199-222

24. Dittmer J C and Lester R L 1964 J. Lipid Res. 5126

25. McClare C W F 1971 Anal. Biochem. 39527

26. Killian J A, Prasad K U, Hains D and Urry D W 1988 Biochemistry 274848

27. LoGrasso P V, Moll F and Cross T A 1988 Biophys. J. 54259

28. Lakowicz J R 2006 Principles of fluorescence spectroscopy (New York: Springer)

29. Chen G C and Yang J T 1977 Anal. Lett. 101195

30. Townsley L E, Tucker W A, Sham S and Hinton J F 2001 Biochemistry 4011676

31. Camaleno-Delango J-M, Zhao X K and Fendler J H 1990 Can. J. Chem. 68888

32. Kemp G, Jacobson K A and Wenner C E 1972 Biochim. Biophys. Acta 255493

33. Veatch W R, Mathies R, Eisenberg M and Stryer L 1975 J. Mol. Biol. 9975 